\title{
Quantitative Sonographic Parameters as a Means of Distinguishing Breast Cancers from Benign Solid Breast Masses
}

\author{
Dorit D. Adler, MD, Donna L. Hyde, BS, Debra M. Ikeda, MD
}

Ultrasound examinations of solid breast masses were reviewed retrospectively to determine whether quantitative data of their dimensions could distinguish benign from malignant tumors. Forty-nine fibroadenomas and 30 carcinomas with cytologic or histologic proof were identified. No significant difference was found between carcinomas and fibroadenomas when comparing the average ratio of length to anteroposterior $(\mathrm{L} / \mathrm{AP})$ diameter or the mean ratio of anteroposterior diameter to transverse diameter $(\mathrm{AP} / \mathrm{T})$. In this limited series, quantification of the degree of mass elongation along the natural breast tissue planes had low sensitivity in distinguishing malignant from benign tumors.
$\mathrm{E}$ arly detection of breast cancer has been correlated with improved survival rates. As small tumor size is one of the predictors of a good prognosis, attempts have been made to improve early detection methods. Palpation of a mass depends on a relatively large tumor size, is greatly influenced by the experience of the examiner, and does not yield definitive information about the composition of the mass. Mammography has proved to be the best means of early detection of nonpalpable breast cancer, but it uses ionizing radiation and results in true-positive rates of only $80-85 \% .{ }^{1}$ Ultrasound is an important adjunct to mammography, its primary role being to distinguish cystic from solid masses. ${ }^{2-4}$ The gray-scale features of solid masses thus far have not proved to be sufficiently specific to allow differentiation of benign from malignant solid masses. ${ }^{4-8}$

Previous sonographic descriptions of solid breast masses usually have been subjective evaluations of echogenicity, shape, and marginal features, without

Received January 2, 1991, from the University of Michigan Hospitals, Department of Radiology, Ann Arbor, Michigan. Revised manuscript accepted for publication March 4, 1991.

Address correspondence and reprint requests to $\mathrm{Dr}$. Dorit D. Adler: University of Michigan Hospitals, Department of Radiology, $1500 \mathrm{E}$. Medical Center Drive, TC2910, Ann Arbor, MI 48109-0326. further quantification. To increase the predictive value of sonography in differentiating benign from malignant solid breast tumors, a limited number of recent reports have analyzed quantitative data, ${ }^{9-11}$ suggesting that certain ratios of tumor length, width, and depth may provide improved sensitivity and specificity in distinguishing benign from malignant breast masses. Because such measurements might prove to be an important diagnostic and clinical aid, we performed a retrospective study of solid breast masses seen on sonography to determine whether such quantitative data could differentiate benign from malignant masses.

\section{PATIENTS AND METHODS}

Results of breast ultrasound examinations performed on women at the University of Michigan Hospitals between 1984 and 1990 were reviewed to identify patients with discrete solid tumors seen on sonography. Only tumors with correlative cytologic or histologic data were included.

Seventy-five women with 79 solid breast masses were identified and are included in this series. Two women each had two discrete carcinomas occurring in the same breast, one woman had two fibroadenomas 
in one breast, and another had bilateral fibroadenomas. The women ranged in age from 20 to 88 years (mean, 52 years). Most tumors (70) were scanned using a 7.5 MHz linear probe, Hitachi 310-EUB (Tokyo, Japan), whereas the remaining 9 masses were studied with a 5 $\mathrm{MHz}$ linear transducer, Acuson 128 (Mountain View, CA). All ultrasound studies were performed prior to any invasive procedures. The length, anteroposterior (AP) diameter, and width were measured retrospectively from the hard copy images by a method described previously. ${ }^{9}$ The masses were also designated as having echogenicity less than (hypoechoic) or greater than (hyperechoic) the surrounding tissue. Also noted were location of the lesion in the breast, whether or not the mass was palpable, and visibility of the lesion on mammography.

The only benign masses included in the study were those specifically diagnosed as fibroadenomas. All lesions were confirmed pathologically (58) or cytologically (21). Thirty masses proved to be malignant, whereas the remaining 49 were fibroadenomas.

\section{RESULTS}

Most masses were located in the upper outer quadrant (35\%), $11 \%$ were in the lower outer quadrant, and $14 \%$ were located in the outer positions of the breast $(9$ o'clock right, 3 o'clock left). Fewer lesions were located in the inner regions of the breast: $14 \%$ occurring in the upper inner quadrant, $8 \%$ in the lower inner quadrant, and $6 \%$ in the inner positions ( 3 o'clock right, 9 o'clock left). Eight percent of all lesions were found in the 12 o'clock position, $2 \%$ were in the $60^{\prime}$ clock position, and only $1 \%$ were retroareolar.

Sixty-one percent of the masses $(48 / 79)$ were palpable (24/49 fibroadenomas, 24/30 carcinomas), whereas the remaining $39 \%(31 / 79)$ were not. Ninety percent $(71 / 79)$ of all lesions could be seen on both mammography and ultrasound, but $10 \%(8 / 79)$ were visualized only by sonography, including one mass in a young (29 year old) woman who did not have a mammogram. All masses were hypoechoic. Eighty-six percent $(42 / 49)$ of fibroadenomas and $97 \%(29 / 30)$ of cancers were visualized on mammography.
The average length $(\mathrm{L})$ of all the masses was $2.1 \mathrm{~cm}$ $\pm 1.0 \mathrm{~cm}$ (range, $0.7-5.0 \mathrm{~cm}$ ). The average transverse (T) diameter was $2.0 \mathrm{~cm} \pm 0.9 \mathrm{~cm}$ (range, $0.8-4.9 \mathrm{~cm}$ ), whereas the mean AP diameter was $1.1 \mathrm{~cm} \pm 0.6$ (range, 0.4-3.6 cm). The diameters for the fibroadenomas and carcinomas are summarized in Table 1.

Calculated ratios included length-to-AP dimensions $(\mathrm{L} / \mathrm{AP})$, and $A P-$ to-transverse diameters $(\mathrm{AP} / \mathrm{T})$. The $\mathrm{AP} / \mathrm{T}$ ratio has also been described previously as height-to-width ratio. ${ }^{9}$ The mean L/AP for all masses was $2.1 \pm 0.8$ (range, 0.7 to 4.0 ) and the mean AP/T was $0.6 \pm 0.2$ (range, 0.2 to 1.2). Ratios for the fibroadenomas and carcinomas are listed in Table 2.

\section{DISCUSSION}

The value of ultrasound in distinguishing a cystic from a solid mass has been documented in numerous studies. ${ }^{2-4}$ Sonographic differentiation of benign from malignant solid breast tumors generally has not been possible with a good degree of accuracy, mainly because of their overlap in gray-scale features, ${ }^{4-8} \mathrm{Al}-$ though previous reports have focused on qualitative features to identify cancerous lesions on ultrasound, more recent studies attempt to utilize quantitative analysis of the sonographic data to allow differentiation of malignant from benign masses. ${ }^{9-11}$

Some authors have suggested that the shape of benign and malignant lesions differs ${ }^{5,9,12}$ Fornage et

Table 2: Comparison of Length/ Anteroposterior (L/AP) and Anteroposterior/Transverse (AP/T) Ratios For Fibroadenomas and Carcinomas

\begin{tabular}{lcc}
\hline & \multicolumn{2}{c}{ Ratios } \\
\cline { 2 - 3 } & L/AP (SD) & AP/T (SD) \\
\hline Fibroadenomas & $2.2 \pm 0.8$ & $0.5 \pm 0.2$ \\
Carcinomas & $1.8 \pm 0.6$ & $0.7 \pm 0.2$ \\
Range of Overlap & $1.4-2.4$ & $0.5-0.7$ \\
\hline
\end{tabular}

SD, Standard deviation.

Table 1: Comparison of Diameters of Fibroadenomas and Carcinomas

\begin{tabular}{|c|c|c|c|c|}
\hline \multirow[b]{2}{*}{ Fibroadenomas $(n=49)$} & \multicolumn{2}{|c|}{ Mean Diameter (cm) } & \multirow{2}{*}{$\begin{array}{c}\text { Standard Deviation (cm) } \\
\pm 0.7\end{array}$} & \multirow{2}{*}{$\frac{\text { Range }(\mathrm{cm})}{0.7-3.9}$} \\
\hline & Length & 1.9 & & \\
\hline & Transverse & 1.8 & \pm 0.7 & $0.8-4.3$ \\
\hline & AP & 0.9 & \pm 0.3 & $0.4-2.0$ \\
\hline \multirow[t]{3}{*}{ Carcinomas $(n=30)$} & Length & 2.5 & \pm 1.2 & $0.8-5.0$ \\
\hline & Transverse & 2,4 & \pm 1.1 & $0.8-4.9$ \\
\hline & AP & 1.5 & \pm 0.8 & $0.4-3.6$ \\
\hline
\end{tabular}


al have postulated that fibroadenomas tend to be longer than they are deep because they grow along the breast's natural tissue planes. ${ }^{9,10}$ These investigators compared L/AP ratios for 49 carcinomas with those of a matched group of 49 fibroadenomas, all with a calculated volume less than $1 \mathrm{~cm}^{3}$ each. ${ }^{10}$ They found that $61 \%$ of carcinomas had an L/AP ratio less than or equal to 1.0 , compared with only $4 \%$ of fibroadenomas.

Nishimura et al stated that breast cancers appear smaller in width on ultrasound than their true size because the invasive component does not adequately reflect the ultrasonic waves and consequently is not seen on the sonographic image. ${ }^{11}$ These investigators noted that owing to the nature of sonography and the structural characteristics of the carcinoma, the lesion also will appear smaller in the anteroposterior dimension, but to a lesser degree than for the width. Nishimura et al found that the ratio of height to width (AP/ T) was greater for 68 malignant lesions than for 30 unspecified benign lesions.

In our own study group, we initially analyzed only those lesions $(\mathrm{N}=25)$ with a volume less than $1 \mathrm{~cm} .{ }^{3}$ Using Fornage et al's initial assumption that an L/AP ratio of less than 1.4 was indicative of cancer and that a ratio greater than 1.4 would likely represent a fibroadenoma, ${ }^{9}$ only 2 of $5(40 \%)$ known cancers and 16 of $20(80 \%)$ known fibroadenomas would have been diagnosed correctly in our series. Thus, using the criterion stated earlier, the sensitivity for detecting cancer was $40 \%$ and specificity was $80 \%$. If a value of 1.0 rather than 1.4 was used (as in a later study by Fornage et al), ${ }^{10}$ the sensitivity for detection of carcinoma was zero.

Because our preliminary data revealed no relationship between the L/AP ratio of a lesion and its volume, we did not further restrict our study to lesions of a particular size. When considering all lesions, irrespective of volume, and using the threshold ratio of 1.4, we found that only $10 / 30$ cancers (33\%) could be diagnosed correctly as malignant although $90 \%$ of fibroadenomas (44/49) could be diagnosed correctly as benign. The sensitivity and specificity for detecting cancer were $33 \%$ and $90 \%$, respectively. Using a cutoff of 1.0 , the sensitivity and specificity were $3 \%$ and $98 \%$, respectively. Thus, although fibroadenomas tended to be longer than they were deep, the difference was not significant enough to allow benign and malignant lesions to be differentiated on the basis of the L/AP ratio in our patients.

To compare our data to that of Nishimura et al, ${ }^{11}$ we calculated the $A P / T$ ratio for all masses. Using the suggested ratio value of 0.8 or greater as a determinant of a malignant lesion, we would have diagnosed correctly only 10 of 30 (33\%) known cancers. Assuming that those lesions with a ratio of less than 0.8 were benign, we were able to distinguish 46 of 49 (94\%) known fibroadenomas as benign. In our patients, the sensitivity of this means of identifying cancer was $33 \%$, and the specificity was $94 \%$. Although our specificity is greater than the $70 \%$ specificity reported previously, our sensitivity was much less than the $72 \%$ sensitivity of Nishimura et al. ${ }^{1 t}$

The degree of error involved in obtaining the numerical ratio limits the value of these classifications. The nature of the sonographic image resolution limits the specificity of the numerator and denominator each to $\pm 1 \mathrm{~mm}$. Because ultrasound examinations are highly operator dependent, such measurements may be erroneous if the lesion is not imaged along its largest diameter or if the transducer is not properly positioned in the longitudinal and transverse planes. The characteristics of the lesions additionally introduce a degree of error if there is posterior shadowing and the lesion has irregular borders, as these make the lesions more difficult to measure. In our series, the variation introduced in the actual measurement of the diameters was minimal, as all measurements were made by one viewer $(\mathrm{DH})$. If, however, these measurements were to be recorded as standard practice in a clinical setting, variability would be introduced owing to subjective variation among viewers. When measuring lesions less than $1 \mathrm{~cm}$ in diameter, the error might be significant and, when further combined in a ratio, would result in an even larger degree of error. Consequently, if the standard use of ratios is to be of clinical value in distinguishing benign from malignant lesions, the ratios would have to differ to a very large degree to compensate for the errors inherent in determining such ratios.

Similar to the previous studies, ${ }^{9,10}$ we found a slight tendency for the L/AP ratio to be larger for fibroadenomas than for cancers. Analysis of the degree of anteroposterior growth compared to transverse elongation for fibroadenomas showed an average that was slightly lower than for carcinomas. Unlike prior studies, however, our data indicated no significant difference in discriminating malignant from benign masses, even when comparing lesions of similar dimensions. Classifying solid masses on the basis of these ratios as benign or malignant resulted in low sensitivities.

Our own study, as well as those cited previously, is limited by the relatively small number of lesions examined. Further investigations using larger numbers of benign and malignant breast masses may provide meaningful data. In conclusion, results from our patient population showed no significant difference between the $\mathrm{L} / \mathrm{AP}$ and $\mathrm{AP} / \mathrm{T}$ ratios for fibroadenomas as compared with carcinomas. The use of these ratios did not prove to be of additional value in the differentiation of benign from malignant solid breast tumors. 


\section{REFERENCES}

1. Moskowitz M: Predictive value, sensitivity, and specificity: Applied epidemiology for the practicing radiologist. In: Moskowitz M (ed): Diagnostic Categorical Course in Breast Imaging. Oak Brook, IL, RSNA, 1986, p 49

2. Jellins J, Kossoff G, Reve TS: Detection and classification of liquid-filled masses in the breast by gray scale echography. Radiology 125:205, 1977

3. Sickles EA, Filly RA, Callen PW: Benign breast lesions: Ultrasound detection and diagnosis, Radiology 151:467, 1984

4. Jackson VP: The role of US in breast imaging. Radiology $177: 305,1990$

5. Cole-Beuglet $C$, Soriano RZ, Kurtz AB, et al: Fibroadenoma of the breast: Sonomammography correlated with pathology in 122 patients. AJR 40:369, 1983

6. Heywang SH, Lipsit ER, Glassman LM, et al: Specificity of ultrasonography in the diagnosis of benign breast masses. J Ultrasound Med 3:453, 1984

7. Jackson VP, Rothschild PA, Kreipke DL, et al: The spectrum of fibroadenoma of the breast. Invest Radiol 21:34, 1986

8. Cole-Beuglet $\mathrm{C}$, Soriano $\mathrm{RZ}$, Kurtz AB, et al: Ultrasound analysis of 104 primary breast carcinomas classified according to histopathologic type. Radiology 147:191, 1983

9. Fornage BD, Lorigan JG, Andry E: Fibroadenoma of the breast: Sonographic appearance. Radiology 172:671, 1989

10. Fornage BD, Sneige N, Faroux MJ, et al: Sonographic appearance and ultrasound-guided fine-needle aspiration biopsy of breast carcinomas smaller than $1 \mathrm{~cm}^{3}$ J Ultrasound Med 9:559, 1990

11. Nishimura S, Matsusue S, Koizumi S, et al: Size of breast cancer on ultrasonography, cut-surface of resected specimen, and palpation. Ultrasound Med Biol 14:139, 1988

12. Kasumi F, Fukami A, Kuno $K$, et al: Characteristic echographic features of circumscribed cancer. Ultrasound Med Biol 8:369, 1982 